\title{
Oral Lesions in Patients Attending Dermatology and STD/HIV Outpatient Department: Analysis of A Cohort of 300 Cases
}

\author{
Dr Anand J. Asia ${ }^{1}$, Dr Girishkumar R. Ambade ${ }^{2}$, Dr Keshav Pagrut ${ }^{3}$ \\ ${ }^{1}$ Professor, Department of Skin \& VD, Government Medical College, Akola, Maharashtra, India \\ ${ }^{2}$ Assistant Professor, Department of Skin \& VD, Government Medical College, Akola, Maharashtra, India \\ ${ }^{3}$ Assistant Professor, Department Of Pathology, Vasantrao Naik Government Medical College, Yeotmal, \\ Maharashtra, India
}

\begin{abstract}
:
Background: The oral mucosa and tongue constitute a barometer, a mirror reflecting the state of health of patient. Similar to skin, lesions in mouth speak for itself. They often provide unique opportunity to understand systemic disease process. With careful and judicious practice many systemic diseases can be identified through the manifestations present in oral cavity. It is in light of this that it was thought proper to carry out a prevalence study of some of commonly occurring oral lesions in a skin, STD/HIV and leprosy OPD.

Aims and Objectives : This study was undertaken to find the prevalence of oral lesions related to dermatoses, sexually transmitted diseases (STD) and Human Immunodeficiency Virus (HIV) infection in outdoor patient department $(O P D)$ with reference to number of cases, age and sex distribution.To determine percentage of symptomatic and asymptomatic patients among those with oral lesions.To analyze into various groups the oral pathologic conditions.To determine the local co-factors for oral lesions, if any.

Methodology: The study was carried out in a cohort of 300 new patients attending the Skin, STD and HIV OPD of a teaching hospital in Department of Skin and V.D. in a Tertiary care centre. All new patients attending the $O P D$, irrespective of age, sex and presenting complaints were entered in the study.

Results: Out of 300 patients studied $183(61 \%)$ were male and 117 (39\%) were females. Majority of patients belonged to age group 20 to 40 years. Out of 116 patients found to be having oral lesions only 15 (13\%) had oral symptoms. $116(38 \%)$ were having oral lesions of which $82(27 \%)$ were males and $34(11 \%)$ were females. Pigmentary disorders, carries and white deposits like candidiasis were much more common in males whereas lichen planus was more common in females.
\end{abstract}

Keywords: Lichen planus, Oral lesions, Pigmentary disorders,

\section{Introduction}

Skin is an important index of health. There are many points of similarities as well as differences between the two protective coverings viz. the skin and mucous membrane. Both may show inflammation, show allergies, may be damaged by systemic infections and diseases. Darrier said that "lesions are the alphabet without which nobody can read the language of skin" [1]. Oral lesions may precede, or follow cutaneous disease or occur together. Many a times they may constitute the only manifestation. In eruptive diseases like drug eruptions and lichen planus, lesions are identical to those found in skin, but the constant maceration, heat, moisture and dissolving action of saliva alter the appearance of eruptions. Many patients attending Dermatology OPD have physiological oral conditions like Fordyce spots, leucoedema, cheek biting, asymptomatic oral conditions like candidiasis, pigmentation, leukoplakia, lichen planus or may have symptomatic oral diseases like aphthous ulcers. Patients may be unaware of the probable interrelationships between presenting cutaneous symptoms and oral disease. Oral lesions may be the primary and only sign of various mucocutaneous diseases. Skin lesions many times strongly associated with oral lesions and could easily be neglected by dentists. It is in this context that the field of diagnostic stomatology has a special role to play. In a way, the oral mucosa is a mirror that reflects the disease process occurring elsewhere and enables one to look beyond what the eyes can merely see.

Oral cavity may be affected by wide variety of diseases ranging from bacterial, viral \& fungal infections to eczematous and immunological conditions like collagen vascular diseases like lichen planus, pemphigus etc, premalignant conditions like leukoplakia to florid malignancy. Although, sexually transmitted diseases (STD) are very rare to find with oral presentations, conditions related to HIV infections often are noticed.

\section{Materials and methods}

The study was carried out in a cohort of 300 new patients attending the Skin, STD and HIV OPD of a teaching hospital in a skin, STD/HIV and leprosy OPD. All new patients attending the OPD, irrespective of age, 
sex and presenting complaints were entered in the study. Referred patients from other departments both at OPD and indoor levels were excluded to avoid bias.

Data was entered into the prescribed proforma and a detailed history including addiction and sexual exposure was noted. Care of oral hygiene was inquired and recorded.

A thorough clinical examination of the oral cavity included examination of lips, teeth, gums, buccal mucosa and hard palate. In patients with oral disorders other mucosal regions like conjunctiva, genital and anus were examined. All patients with oral lesions were examined to rule out systemic involvement.

\section{Following investigations were done whenever needed:}

1. $10 \% \mathrm{KOH}$ mount was done in all cases with coating and white deposits on tongue or oral cavity.

2. Tzanck smear was done in patients with vesicobullous lesions.

3. Serum HIV and VDRL were done in patients having history of sexual exposure and blood transfusion.

4. Oral and skin biopsies were advised when diagnosis could not be established clinically.

\section{Observations and Results}

1) Age group and sex distribution: Out of 300 patients studied $183(61 \%)$ were male and $117(39 \%)$ were females. Majority of patients belonged to age group 20 to 40 years.

2) Employment status: Out of 300 patients 129 (43\%) were employed, of which $54(18 \%)$ were skilled labourers, 75 (25\%) were unskilled labourers while 171 (57\%) were unemployed.

3) Prevalence of addiction: Total numbers of persons addicted to some or other substances were 114 $(38 \%)$. Males were $83(72 \%)$. Maximum numbers of persons addicted were in age group 21-50 years. Smoking (28.6\%) was the commonest addiction in males.

4) Symptomatology of oral lesions: Out of 116 patients found to be having oral lesions only 15 (13\%) had oral symptoms.

5) Oral diseases and sex groups: Out of 300 patients $116(38 \%)$ were having oral lesions of which 82 $(27 \%)$ were males and $34(11 \%)$ were females. Pigmentary disorders, carries and white deposits like candidiasis were much more common in males whereas lichen planus was more common in females.

6) Sites of involvement: Majority of patients had involvement of buccal mucosa and teeth. Multiple site involvement like buccal mucosa, gingiva and teeth were observed in 24 (20\%) patients.

7) Oral diseases and addiction: Out of 116 patients having oral lesion 65 (56\%) patients were addicted to some or other substances. Hyperpigmentation of oral mucosa was the commonest observation in $20(80 \%)$ patients who had addiction. Lichen planus did not show any relation to addiction.

8) HIV and oral diseases: Total 19 patients were HIV seropositive and 12 (63\%) of them had oral lesions. Oral candidiasis was the commonest manifestation observed in $6(50 \%)$ patients.

9) Clinical presentation: Out of 300 patients, 296 (98.6\%) had cutaneous diseases. Cutaneous as well as oral diseases were observed in 113 (37\%) patients. Out of 296 patients with skin diseases majority of patients had ezema, bacterial and fungal infections. Lichen planus was seen in 8 (3\%) patients.

\section{Discussion}

Oral lesions were more commonly seen in third and fourth decades, maximum being males (45\%). This may be because of more males attending OPD and more prevalence of addiction in them (80\%). Pigmentation of mucosa was found in elderly persons whereas caries was found in children and young individuals. The pattern of cutaneous manifestations varied in different age groups. Bacterial and fungal infections were more common in children and adults while eczema was more common in elderly. Pediatric age group constituted 5\% of the cohort which corroborates well with Klienman's [3] observation of $4 \%$.

In this cohort, $129(43 \%)$ were employed and 177 (57\%) were unemployed. Among employed 54 $(18 \%)$ were skilled labourers while $75(25 \%)$ were unskilled labourers. Majority of males (80\%) had addiction and smoking was the commonest addiction. Other common substances were tobacco, betel leaves and areca nuts. Kleinman has observed addiction to tobacco in $10 \%$ of young patients which was $7 \%$ in our study [3].

Sixty three percent of patients used toothbrush to clean their teeth while finger and datoon were used by $37 \%$ of patients. This may be due to lower socioeconomic status and immigrant population from villages. Different teeth cleaning habits did not have any significance.

Prevalence of oral diseases was $38 \%$ and most common conditions were pigmentation and staining of teeth. Caries, Fordyce spots, and oral candidiasis were observed in $55 \%$ of patients. Lichen planus accounted for $5 \%$ while ulcerative lesions like pemphigus and aphthous ulcers were seen in 9\%. Out of 116 patients who had oral lesions, only $15(13 \%)$ were symptomatic and pain was the commonest symptom. Common symptomatic diseases were oral candidiasis, ulcerative diseases like pemphigus vulgaris, aphthous ulcers and herpes zoster. Most common site of involvement was buccal mucosa (38\%). Involvement of multiple sites like lips, gums, teeth and buccal cavity was seen in pigmentary disorders, drug reaction and ulcerative diseases. 
Pigmentation abnormalities were observed in $25(8.6 \%)$ of which majority were males (80\%). This may be because of more substance abuse in males. The reported prevalence of pigmentary disorders in general population is $12.7 \%$ [4]. Out of 8 patients with lichen planus, $6(75 \%)$ had oral involvement. Sixty six percent were females which is similar to finding of Daftary DK who reported higher prevalence of oral lichen planus in females as compared to males [5]. There is no direct association between tobacco use and oral lichen planus observed by Gupta PC as well [6]. Oral candidiasis was observed in $12.9 \%$ of patients in our study which is similar to observations of $10 \%$ by Driesen [7]. The marginal higher percentage in our study may be due to inclusion of HIV seropositive patients. Erosive and ulcerative diseases were found in 11 (9.4\%) of patients. In our study, all patients of pemphigus vulgaris had oral lesions and prevalence of oral lesions in pemphigus is $60 \%$ [8]. Herpes zoster, Steven Johnson syndrome and discoid lupus erythematosus (DLE) were some of other ulcerative diseases seen. There is varied prevalence of depigmented lesions like leukoplakia, DLE, and vitiligo observed by Ikeda (1.1\%) and by Macigo (10.6\%) whereas it was seen in only $0.3 \%$ patients in our study [9]. Out of 19 HIV seropositive patients, oral lesions were found in $12(63 \%)$ cases. Oral candidiasis was the commonest manifestation seen in 50\% of patients which is similar to reported by Legott [10]. However, prevalence of oral candidiasis in HIV seropositive patients ranges from $50 \%$ to $80 \%$ as observed by Wanzala and Bertazoli $[11,12]$.

\section{References}

[1]. Samhit MH et al. Some dermatological aspects of oral medicine. Oral Surg Oral Med Oral Pathol 1967 Dec; $24: 746$

[2]. Cruz GD. Accurate diagnosis of oral lesions in HIV. Arch otolaryngol Head and Neck surg 1996; 122 (1): $68-73$.

[3]. Kleinman DV, Swango PA, Pindborg JJ. Community epidemiol 1994; 22(4): 243.

[4]. Macigo FG, Mwaniki DL, Prevalence of oral mucosal lesions in Kenyan population. East African Med Journal 1995 Dec; 72 (12): 778 .

[5]. Daftary DR, Bhansale RB, Murti RB. Scan J Dental Research 1998 June; 88 (3): 224

[6]. Gupta PC, Murti PR, Bhosale PR, Mehta FS. Effect of cessation of tobacco use on the incidence of oral mucosal lesions. Oral diseases. 1995 March; 1 (3): 54-58.

[7].
[8].

[9]. Ikeda N, Handa Y, Khim SP, Durriward C. Prevalence study of oral mucosal lesions in a selected combodian population. Community Dent Oral Epidemiol 1995 Feb; 23 (1): 49-55.

[10]. Legott PJ, Mastrucci. Oral lesions in child with HIV. Int Cnf AIDS 1990 July; 20-23; 6(1):216.

[11]. Wanzala P, Manzi F.Low prevalence of oral mucosal lesions in HIV seropositive women. J Oral Path Med 1989 ; $18 ; 416$.

[12]. Bertozzoli R, Lourenco. Oral manifestation of HIV infection. Int conf of AIDS 1994; 7: 184. 